\title{
Remesas y desarrollo humano en San José Chiltepec, Oaxaca. Un estudio a partir del enfoque de capacidades
}

\author{
Remittances and human development in San José \\ Chiltepec, Oaxaca. A study from the capability approach
}

doi: http://dx.doi.org/10.32870/

espiral.v23i66.5302

\begin{abstract}
Resumen
En este trabajo se presentan los resultados de una investigación llevada a cabo para establecer la relación e incidencia que tienen las remesas en el desarrollo humano en el municipio de San José Chiltepec, Oaxaca. Este examen se realizó a partir de un estudio de percepción que se fundamentó en el enfoque de capacidades formulado por Amartya Sen. Los resultados obtenidos muestran que los beneficios que propicia la migración, de los cuales dan cuenta los informes mundiales de desarrollo humano del 2007 y 2009 , no son tan significativos pues las remesas son utilizadas casi en su totalidad para financiar el consumo. En este sentido, el potencial para que los sujetos puedan transformar sus circunstancias de vida es limitado, ya que no incrementa de manera sustancial los funcionamientos y las capacidades de quienes las reciben, afectando su libertad y capacidad de acción.
\end{abstract}

Palabras clave: remesas, desarrollo humano, enfoque de capacidades, funcionamientos, bienestar.
Joel Ruiz Sánchez

Beatriz Acosta Uribe**

\begin{abstract}
In this study, we present the results of research carried out to establish the relation and incidence of remittances on human development in the town of San José Chiltepec, Oaxaca. This study was developed from a study of perception based on the Capability Approach formulated by Amartya Sen. The results obtained show that the benefits of migration which cover Human Development Reports of 2007 and 2009, are not significant, because remittances are used almost entirely to finance consumption. In this respect, the potential of subjects to transform their living circumstances are limited: it does not change substantially functions and capabilities in recipients, affecting their freedom and action capacity.
\end{abstract}

Keywords: Remittances, human development, capability approach, functions, welfare.

-Profesor-Investigador de la Facultad de Estudios Sociales de la Universidad Autónoma del Estado de Morelos. jorsan30@hotmail.com

-•Profesora-Investigadora de la Universidad del Papaloapan, campus Tuxtepec.

acurbe@hotmail.com 


\section{Introducción}

Poco se sabe, en general, sobre el efecto de las remesas sobre la calidad de vida de las personas en los términos en que la conciben los enfoques de capacidades y del desarrollo humano con base en un estudio de percepción. La literatura sobre el tema ha observado que las familias cuya principal fuente de ingresos es el dinero por concepto de remesas construyen su casa, adquieren bienes de consumo duradero, financian la fiesta del pueblo o lo emplean para organizar alguna celebración familiar. Sin embargo, no se sabe si su calidad de vida mejora, entendido este concepto como una idea global del desarrollo humano de una persona, más allá del incremento en el consumo.

Razonablemente, se podría argumentar que al menos algunos utilizan el dinero para invertirlo en rubros distintos a los mencionados, que incluirían el mejoramiento en su cuidado médico o el financiamiento de los estudios de los hijos, así como gastos de recreación en general, aunque, como lo veremos más adelante, esto no siempre es así. En casos muy concretos, la cantidad de remesas podría estar asociada con una mejor calidad de vida y un aumento de la libertad, como lo señala Sen (1985).

Es cierto que lo anterior puede ser un ejercicio de libertad, pero también resultado de la falta de opciones y, en ese sentido, puede ser una restricción en la libertad como consecuencia de un proceso de exclusión en los países de origen. Sin embargo, no existen datos suficientes, ni desde un enfoque teórico-metodológico centrado en el sujeto, el cual permita adentrarnos en este asunto y corroborar también si se cumplen estas condiciones que señala la perspectiva del desarrollo humano.

Es por ello que en este trabajo se realiza un análisis de los resultados que arrojó la aplicación de un instrumento diseñado a priori para estudiar la relación entre las reme- 
sas y el desarrollo humano en San José Chiltepec, Oaxaca, municipio con altos grados de pobreza y marginación, así como una tradición emergente de migración a los Estados Unidos. Los resultados muestran, por lo menos para el caso de estudio, que las remesas son utilizadas casi en su totalidad para financiar el consumo, y en este sentido el potencial para que los sujetos puedan transformar sus circunstancias de vida es limitado, con lo cual se contradice lo que establecen los citados informes de desarrollo humano cuando sostienen que las remesas mejoran el nivel de desarrollo humano de las personas ligadas con la migración.

\section{Migración, remesas y desarrollo humano}

El Programa de las Naciones Unidas para el Desarrollo (PNUD) comenzó a difundir y utilizar el concepto desarrollo humano en 1990. En su primer informe, elaborado ese mismo año, lo definió como un proceso de ampliación de las opciones de las que disponen los individuos (PNUD, 1990), en el cual se contemplan tres grandes tópicos para alcanzar un nivel de vida decoroso y digno para las personas: a) la posibilidad de disfrutar de buena salud; b) el acceso a la educación; y c) la capacidad de generar ingresos para una mejor calidad de vida.

De acuerdo con el Informe sobre Desarrollo Humano 2007 (PNUD, 2007), el desarrollo humano es la expansión de la libertad de las personas. La libertad es el conjunto de oportunidades para ser y actuar, y la posibilidad de elegir con autonomía. En relación a la migración, el citado programa establece que la movilidad humana forma parte de la libertad que gozan las personas, pues al migrar están directamente ejerciendo su facultad de decidir, y en ese sentido esa elección es constitutiva del desarrollo humano, aunque se aclara que tal proceso puede estar condicionado por el desigual acceso a oportunidades en las regiones de origen. 
Ahora bien, partiendo de esta perspectiva asumida por el Programa de Naciones Unidas para el Desarrollo, surge la siguiente pregunta: ¿por qué es interesante entender la relación entre las remesas y el grado de desarrollo humano de una comunidad o en el plano individual? Lo primero que hay que señalar al respecto es que la relación entre remesas y desarrollo humano es compleja. Por un lado, si no fuera por el dinero que envían los migrantes a sus familias, las condiciones de pobreza y marginación en muchas regiones de México serían peores. Por otro, la migración genera resquebrajamiento de familias y comunidades. Además, si bien compensan a nivel de hogares, no necesariamente retribuyen la pérdida de potencial de crecimiento de largo plazo en las regiones. Es decir, se pierde capacidad fiscal y productiva a nivel local; entonces, al disminuir estos elementos, las regiones por lo general verán limitado su desarrollo en el mediano y largo plazo.

En este sentido, y a pesar de los beneficios que pudiera traer consigo la migración en los países de origen, existen opiniones encontradas (Moreno Pérez, 2008; García Zamora, 2009) sobre el efecto que genera la ausencia de los familiares migrantes. Aunque una parte de la literatura reconoce los beneficios económicos que en un primer momento pueden tener las remesas para las familias de los migrantes, también se sostiene que tienen un costo que no es propiamente económico y que cruza las áreas de la identidad, la salud, y en general el bienestar físico y emocional, esto no sólo para quien decide partir, el que se va, sino para las familias, que funcionan como redes sociales significativas (Hurtado, et al., 2008). Esposo, padre, madre, hermano, hermana, hijos, incluso amigos, no importa quién decida partir, siempre se genera un desequilibrio en la estructura y funcionalidad de la familia. En este sentido, se reconocen problemas en la salud y en el comportamiento de quienes se quedan (Hurtado, et al., 2008; Aguilar, et al., 2008,), así como redefinición de roles 
sociales y estilos de vida que trastocan la vida familiar de quienes se van, pero también de los que se quedan.

Todos estos elementos inciden de manera significativa en la calidad y el tipo de vida que experimentan el migrante y sus familiares. Al tratar de establecer la relación entre migración, remesas y desarrollo humano, se tienen que tomar en cuenta todos estos aspectos, puesto que este último no involucra solamente las dimensiones económicas o materiales, sino que tiene que ver también con la salud, la educación y en general con el bienestar físico y emocional de las personas. Así pues, las libertades de ser y hacer son los factores que articulan y le dan sentido al enfoque de capacidades formulado por Amartya Sen (1985).

\section{El enfoque de capacidades de Amartya Sen}

A pesar que el pensamiento de Sen adolece de una sistematización adecuada, y de que es fácil perderse entre tantas líneas de investigación que ha generado, se coincide con Cejudo (2007) cuando asevera que el enfoque de capacidades (capabilities approach) proporciona elementos muy interesantes para entender y unificar una buena parte de sus aportes a la filosofía y el pensamiento social contemporáneo.

Para Sen, las capacidades de una persona son fundamentales para acceder a la libertad. De este modo, construyó una teoría de la libertad como capacidad, válida para interpretar las acciones de los sujetos sociales en el contexto de las políticas económicas globales y del sentido que se le ha dado al desarrollo a través de estas (Cejudo, 2007).

El enfoque de capacidades sostiene que el desarrollo no tiene que ver solamente con el crecimiento de la producción económica, y que la forma de medirlo no es únicamente a través de la renta per cápita y el PIB nacionales (Sen, 1985). Para Sen, el desarrollo se relaciona más bien con lo que las personas quieren hacer y ser. El autor conceptualizó las 
ideas anteriores a través de las categorías de funcionamientos y capacidades, vehículos para elegir y poder alcanzar diferentes clases de vida, y con ello configurar una sociedad más libre en la cual el desarrollo sea el motor de la libertad personal y social (Sen, 1985).

No obstante que el enfoque de capacidades fue planteado originalmente por Sen para estudiar y medir la calidad de vida, es indudable que este ha trascendido a otras esferas sociales. Así, se ha convertido en un importante instrumento para analizar y resignificar la idea del bienestar, la pobreza, las acciones sociales y el desarrollo. En ello estriba la importancia y aportaciones del enfoque en cuestión. La lógica empleada por Sen implica pensar los conceptos anteriores desde una nueva perspectiva, la cual pone en el centro del debate la idea de que la calidad de vida depende de lo que los sujetos sean capaces de conseguir, de las maneras en que sean capaces de vivir, y no de la renta, disponibilidad de servicios sociales o la satisfacción de necesidades básicas (Sen, 1985).

El enfoque del desarrollo humano tuvo como punto de partida el enfoque de capacidades elaborado por Amartya Sen. La visión de este se centra en las capacidades y deseos de los sujetos sociales. En este sentido, el desarrollo, partiendo de la visión de Sen, se convierte en un proceso de expansión de las capacidades de cada persona para llevar el tipo de vida que valora (Sen, 1995).

Siguiendo esta línea de pensamiento, los logros en las políticas de desarrollo deben evaluarse en la medida en la que incrementan la participación de los individuos en el proceso de toma de decisiones y las oportunidades para alcanzar lo que se desea (Sen, 1995). Desde esta perspectiva, la pobreza, según el autor, se convierte en una privación de capacidades. ${ }^{1}$

I. En esta definición, Sen incluye aspectos como la mortalidad prematura, las hambrunas, la desnutrición y la falta de libertad para trabajar. Asimismo, están presentes o se consideran la ausencia de libertades políticas y de derechos civiles (Sen, 1995). 
El desarrollo debe constituirse en una especie de proceso participativo en el que los individuos, antes que ser entes pasivos, se erijan en agentes activos de las políticas públicas mediante actos que se despliegan de manera soberana.

Para examinar y medir de manera adecuada el bienestar individual, Sen propone que no solamente se evalúen los recursos de que disponen los sujetos, ya que estos no proporcionan información suficiente y fidedigna, pues la forma en la que se utilizan y aprovechan varía de acuerdo a circunstancias muy específicas como la edad, la salud, la educación y el capital social de las personas (Sen, 1995).

En este tenor, los recursos adquieren valor intrínseco por lo que representan y por su potencial para definir y desarrollar capacidades en los individuos. Sen los consideró en ese sentido, por lo cual los tipificó como oportunidades (entitlements), que son los recursos de que disponen los sujetos en el marco de una estructura de derechos. Una persona está habilitada cuando posee recursos y derechos, los cuales usa para obtener un bien o servicio adicional; puede incluir también la propia fuerza de trabajo (Sen, 1985). Así, las oportunidades son fundamentales para definir capacidades, entendiendo que estas son las cosas que el sujeto hace o puede hacer; dicho de otro modo, se refiere a acciones concretas desplegadas por los individuos, para lo cual el sujeto necesita estar habilitado. Los usos de esas habilitaciones van a determinar el grado de libertad y bienestar de las personas (Sen, 1995).

\section{Indicadores de desarrollo humano y rezago social en la región: el punto de partida}

Para poder realizar un análisis más preciso sobre el impacto e incidencia que han tenido la migración y en particular el envío de remesas en San José Chiltepec desde el punto de vista de las personas involucradas en ella, es 
necesario examinar algunos indicadores oficiales relacionados con el bienestar y la calidad de vida de los habitantes de la comunidad. Esto es importante porque permite contrastar los datos y determinar las implicaciones que tiene el fenómeno de la migración en el desarrollo humano de los familiares de los migrantes. En segundo lugar, sirve para comparar la dimensión objetiva presente en estas cifras oficiales con los resultados de percepción que se obtuvieron mediante la aplicación del instrumento.

En esta tesitura, es de sobra conocido que el estado de Oaxaca presenta diferentes condiciones que han limitado su desarrollo. No se trata de ver el desarrollo únicamente como el crecimiento del PIB por habitante o la inversión productiva, sino que este debe incorporar otras dimensiones que, como la salud y la educación, resultan igualmente importantes para la calidad de vida o el bienestar de las personas. Es en este sentido que utilizamos dicho concepto, es decir, en referencia a las condiciones que históricamente han afectado a la mayor parte de los habitantes del estado de Oaxaca. Con ello no se está diciendo que haya una ausencia de servicios públicos o falta de desarrollo económico, sino más bien se está refiriendo a la mala calidad y la deficiente cobertura y posibilidad de acceso a los mismos.

Del mismo modo, se conjuntan otros fenómenos que generan un escenario propicio para que la migración se desarrolle en su territorio. Dentro de las condiciones que hacen posible la movilidad poblacional en Oaxaca se encuentran, entre otras, la existencia de altas tasas de marginación y pobreza, las cuales tienen en la exclusión su principal razón de ser; la presencia de un importante deterioro en la actividad rural -en donde se ocupa más de la mitad de la población económicamente activa (Acosta Uribe, Ruiz Sánchez, et al., 2009)-; y la falta de empleos bien remunerados, asociados con la baja calificación y altas tasas de analfabetismo. Todos estos son problemas estructurales 
que han definido el perfil socioeconómico del estado y que, se insiste, forman parte de mecanismos de exclusión que históricamente se han configurado en la entidad.

La cuenca del Papaloapan, a pesar de ser la región que más aporta a la economía oaxaqueña, ha experimentado en términos generales el mismo patrón de desarrollo que el resto del estado. A excepción de Tuxtepec, los demás municipios que la conforman son predominantemente rurales, con una tendencia natural a generar condiciones de exclusión, pobreza y marginación entre las personas que en ellos habitan. La región está integrada por veinte municipios, de los cuales once son considerados por el Consejo Nacional de Población (CONAPO, 2005) con grados muy elevados de marginación (Acosta Uribe, Ruiz Sánchez, et al., 2009).

Asimismo, para 2005, la cuenca del Papaloapan registró un porcentaje de pobreza alimentaria del $34 \%$, de capacidades del $43.2 \%$, y de patrimonio del 67.3\% (CONAPO, 2005). Este panorama muestra un contexto regional marcado por grandes carencias y rezagos, que, puestos en perspectiva, explican por sí solos las condiciones de vida de una buena parte de los habitantes de la cuenca oaxaqueña del Papaloapan y por qué la migración se ha convertido en una alternativa para escapar de esas condiciones por demás difíciles.

El porcentaje de población que no tiene la primaria completa ascendió al 40.47\%, lo que confirma una importante pobreza de capacidades (CONAPO, 2005). Esta situación será clave para examinar más adelante si realmente las remesas han modificado este estado de las cosas a partir de que comenzaron a recibirse en esta comunidad, pues hay que recordar que este aspecto es uno de los elementos clave en la perspectiva que plantea el enfoque de capacidades.

En el caso del municipio de San José Chiltepec, este tenía en el 2005 un índice de desarrollo humano de 0.68, y de 0.74 en el 2010. Como se puede apreciar, el incremento en cinco años fue ligeramente a la alza. Aunque estos datos 
permitan clasificar al municipio con un grado de desarrollo humano medio-alto, lo cierto es que se deben tomar estas cifras con reserva, pues, como se ha sostenido en este trabajo, la construcción de los índices de desarrollo humano es poco confiable y no toma en cuenta otros elementos que tienen que ver con el entorno social y las circunstancias de vida de las personas.

De entrada, se podría suponer que este escaso crecimiento tiene relación con la migración en el sentido que un número importante de personas migraron en este periodo, y, por otro lado, que la pobreza no ha disminuido con la llegada de las remesas. Desafortunadamente, este índice de desarrollo humano por municipios no se ha vuelto a realizar, por lo que no se cuenta con estos datos para poder visualizar cuál ha sido su comportamiento en los últimos ocho años.

Independientemente de la limitante que esto representa, lo cierto es que los datos disponibles, y que aquí se presentan, son suficientes para poder realizar un análisis comparativo y poder determinar el impacto de las remesas en el desarrollo humano, así como la influencia que tiene este contexto en la percepción de las personas en relación al uso e impacto de estos recursos en su calidad de vida.

Con respecto a los indicadores de rezago social que elaboraron para el año 2010 los organismos oficiales, las cifras son coincidentes en relación con pobreza, marginación y desarrollo humano. El Cuadro 1 presenta un panorama en este sentido.

En relación a la educación, en el Cuadro 2 se pueden observar algunos datos que muestran las condiciones al respecto, y las tasas de alfabetización en el distrito de Tuxtepec, particularizando el caso de San José Chitepec. Estos datos, que coinciden con los momentos de mayor flujo migratorio en la región, ponen en evidencia el rezago educativo prevaleciente en esta.

\section{8}


Cuadro 1. Indicadores de rezago social en San José Chiltepec (2010)

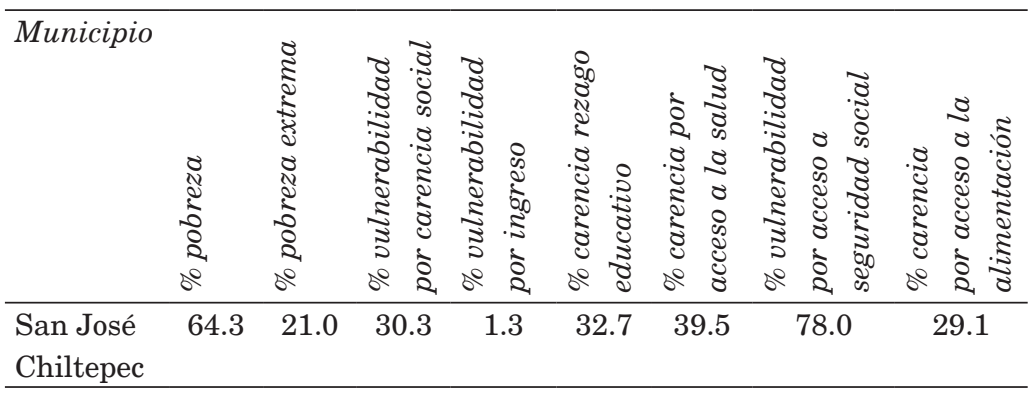

Fuente: cuadro elaborado con datos del CONAPO (2010).

Cuadro 2. Alfabetización en el municipio de San José Chiltepec (2005)

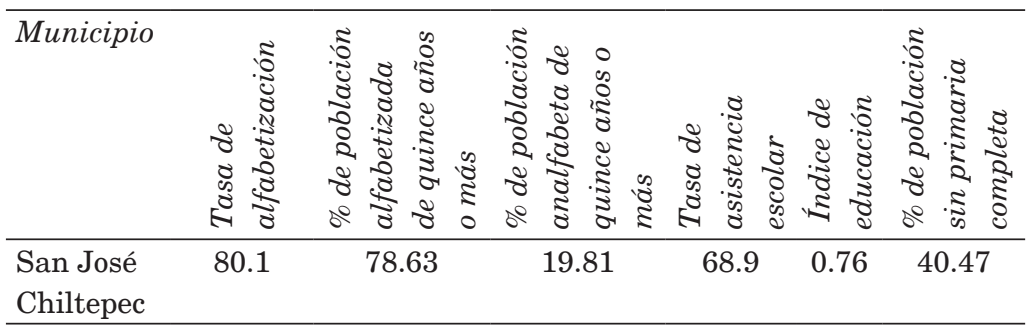

Fuente: cuadro elaborado con datos del CONAPO (2005).

\section{Método}

\section{I. Participantes}

El instrumento fue aplicado a cincuenta y seis personas (dieciséis hombres y cuarenta mujeres) pertenecientes al municipio de San José Chiltepec, Oaxaca, las cuales tenían una edad promedio de cuarenta y tres años al momento de responder el cuestionario. Todos los sujetos habían sido previamente ubicados en función de su parentesco directo 
con los migrantes, es decir, cónyuge, padre o madre (ver Cuadro 3).

Cuadro 3. Promedio de edad de las personas encuestadas

\begin{tabular}{lccc}
\hline Sexo & $\begin{array}{c}\text { Número de } \\
\text { personas }\end{array}$ & Media de edad & $\begin{array}{c}\text { Desviación } \\
\text { estándar de edad }\end{array}$ \\
\hline Hombres & 16 & 38.19 & 13.26 \\
Mujeres & 40 & 44.40 & 16.04 \\
Total & 56 & 42.63 & 13.15 \\
\hline
\end{tabular}

Fuente: elaborado con datos del cuestionario de desarrollo humano (Ruiz y Acosta, 2013).

\section{2. Instrumento}

La obtención de los datos para la evaluación del desarrollo humano se realizó a partir de la elaboración de un instrumento estructurado con formato de respuesta tipo Likert con cuatro opciones de respuestas: totalmente de acuerdo, de acuerdo, en desacuerdo y totalmente en desacuerdo. La escala está compuesta de tres apartados: circunstancias, funcionamientos y capacidades.

La primera dimensión que se analiza es la que tiene que ver con los antecedentes relacionados con las circunstancias de vida antes de recibir las remesas. Este aspecto es importante porque da cuenta de las condiciones de vida en las que estaban inmersos los familiares de los migrantes antes de la partida del familiar y, por consiguiente, de la recepción de las remesas. Asimismo, permite comparar la percepción con base en los recursos recibidos y el impacto que han tenido estos desde la propia visión de los involucrados en la definición de los funcionamientos y las capacidades. De igual forma, se pueden establecer vínculos con los datos oficiales relativos a pobreza, marginación y desarrollo humano que se presentaron en un apartado anterior.

Los informes mundiales de desarrollo humano consideran que la salud y la educación son dos aspectos que 
constituyen una base muy importante para poder alcanzar funcionamientos y capacidades que a su vez permiten a las personas tener una vida plena y duradera, pues sin salud ni educación no se pueden alcanzar las metas y objetivos que cada individuo trace, como decidir la vida que quiere llevar. En este nivel, la libertad asume un rol preponderante, ya que es el punto de partida de las acciones y decisiones que finalmente conducen a la definición de la vida deseable.

Es coincidente en este punto lo planteado en el último informe de la Organización Internacional para las Migraciones (OIM), que sostiene que

[...] el enfoque relativo a las capacidades es un importante adelanto que trasciende la idea convencional del desarrollo centrado en el crecimiento económico [...]. Las libertades y las capacidades, tal como se entienden en los ámbitos de la salud, la educación, y otras esferas semejantes, son indudablemente valiosas por derecho propio, pero también lo son en la medida en que contribuyen a la felicidad. En ese sentido, el debate sobre el bienestar y la felicidad es un elemento pertinente de la agenda del desarrollo (OIM, 2013, p. 89).

La segunda dimensión tiene que ver con los funcionamientos definidos como los logros obtenidos gracias a las remesas, los cuales son evaluados con la percepción de calidad de vida y del uso que se le ha dado a estos recursos.

Finalmente, el tercer apartado hace referencia a las capacidades o habilidades para alcanzar la calidad de vida deseada a través de la posesión de recursos. Este aspecto se determina a partir de la posibilidad de disfrutar de buena salud y del acceso a la educación.

En este sentido, las circunstancias constan de seis reactivos, mientras que los funcionamientos son evaluados con dieciséis y las capacidades con doce. 


\section{3. Conducción del estudio}

El instrumento se aplicó en el mes de julio del 2013. Se realizó una visita domiciliaria a las personas identificadas como familiares de los migrantes en Estados Unidos, se les explicó el objetivo de la investigación y se les solicitó contestar el cuestionario. El encuestador leyó cada una de las preguntas y fue recopilando las respuestas a partir de la información que proporcionaban los familiares de los migrantes. El tiempo promedio que tardaron en responder fue de 15 a 20 minutos.

\section{4. Análisis de la información}

Las puntuaciones directas se transformaron en calificaciones jerárquicas porcentuales, las cuales se basan en la comparación interindividual a partir del rango de calificaciones crudas; ${ }^{2}$ esta comparación indica la posición de una persona en una escala de 101 puntos, donde la calificación más alta asciende hasta 100 y la más baja desciende hasta 0. La fórmula es:

$$
X \%=100 \frac{(X-B)}{(A-B)}
$$

Donde:

$\mathrm{X}=\quad$ cualquiera de las calificaciones crudas.

$\mathrm{B}=\quad$ calificación cruda más baja.

$\mathrm{A}=\quad$ calificación cruda más alta.

$(J R)=$ calificación jerárquica (en esta fórmula califica el porcentaje de calificaciones crudas). 
Cuadro 4. Calificación jerárquica porcentual e indicadores a partir de las puntuaciones obtenidas por los participantes

\begin{tabular}{lc}
\hline Percepción de desarrollo humano & Calificación jerárquica \\
\hline Óptimo & $81-100$ \\
Bueno & $61-80$ \\
Aceptable & $41-60$ \\
Vulnerable & $21-39$ \\
Intolerable & $0-20$ \\
\hline
\end{tabular}

Fuente: Morales (2005).

\section{Resultados}

Los resultados se distribuirán en tres apartados: en el primero se presentarán las calificaciones jerárquicas porcentuales de cada una de las dimensiones; en el segundo se abordarán las comparaciones entre las dimensiones trabajadas, así como la relación entre estas; y en el tercer apartado se encontrarán las diferencias entre hombres y mujeres respecto a su percepción con los indicadores de desarrollo humano.

6.I. Resultados de las calificaciones jerárquicas porcentuales de las dimensiones de desarrollo humano

Como se mencionó anteriormente, una vez capturados y procesados los datos, se generaron las calificaciones crudas, la cuales se convirtieron en calificaciones jerárquicas porcentuales, de tal manera que estas permitieran obtener un indicador sobre la percepción de desarrollo humano.

La Gráfica 1 muestra los resultados del indicador de circunstancias de vida, el cual es definido operacionalmente como el antecedente sobre educación y salud de los familiares de los migrantes. En este sentido se puede apreciar que los encuestados reportaron que antes de recibir las remesas se encontraban en un nivel "bueno" en cuanto a 
la educación (CJP=62) y en un nivel "aceptable" en el rubro de salud (CJP=54).

Gráfica 1. Resultados del indicador de circunstancias de vida

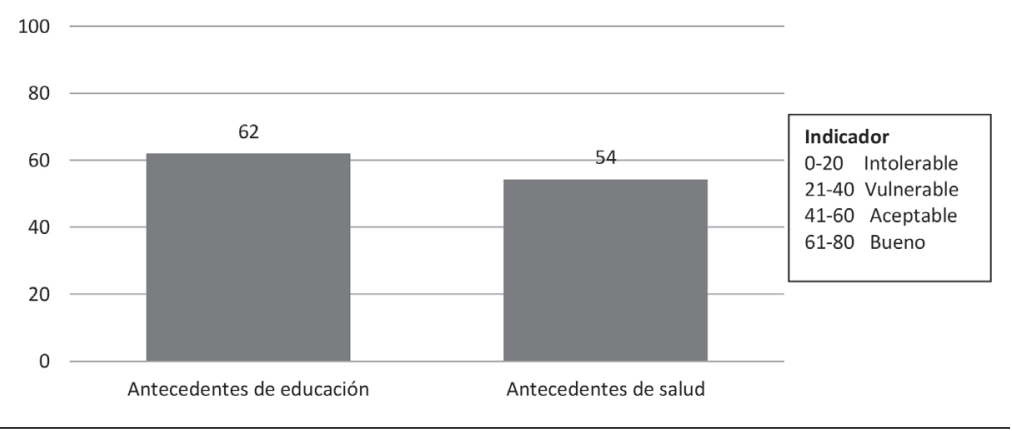

Fuente: elaboración propia.

Como se mencionó anteriormente, las dos dimensiones en cuestión son generadoras de funcionamientos de acuerdo a lo planteado por el propio Sen, pero también por las instancias internacionales encargadas de generar informes de desarrollo humano, como el PNUD. Los resultados nos indican que la percepción de las personas es medianamente positiva en relación a las condiciones de vida que tenían antes de recibir las remesas, sobre la base del acceso a la salud y la educación.

Con respecto a los resultados del indicador de capacidades, el cual evaluó la habilidad que tienen los familiares de los migrantes a partir de la posesión de bienes, y el cual se operacionalizó con base en la comparación realizada en las dimensiones de educación y salud a raíz de la recepción de las remesas, se obtuvo que la comparación en cuanto a la educación se ubicó en un nivel "aceptable" (CJP=56) y el rubro de salud obtuvo un nivel "bueno" (CJP=66) (ver Gráfica 2). 
Gráfica 2. Resultados del indicador de capacidades

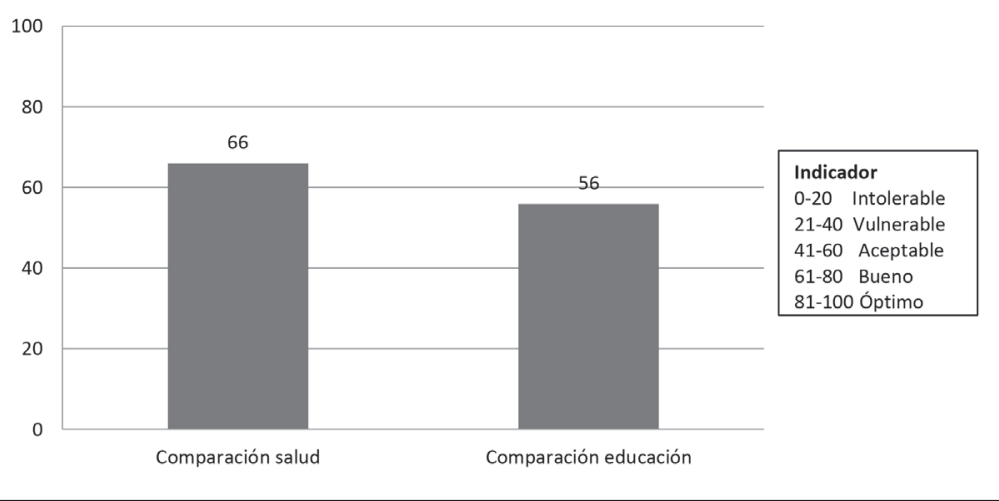

Fuente: elaboración propia.

El indicador funcionamientos tiene que ver con el ingreso, representado en este caso por el uso que se le da a las remesas, y con la percepción que tienen los familiares de los migrantes sobre su calidad de vida. Los resultados reportaron que la percepción sobre la calidad de vida de los familiares de los migrantes se encuentra en un nivel "óptimo" (CJP=88), es decir, existe una percepción global de que las remesas sí impactan de manera positiva la calidad de vida y el bienestar. Este aspecto tiene indudablemente una relación directa con el uso de las remesas en la alimentación, el cual se ubicó en un nivel "bueno" (CJP=78).

La percepción es que las remesas están incidiendo positivamente en el mejoramiento de las condiciones de vida sobre la base de la alimentación, pues permiten garantizar de manera adecuada una necesidad fundamental, sin la cual no es posible acceder a otros bienes y servicios que permitan configurar funcionamientos.

La utilización de las remesas para la compra de bienes muebles, como por ejemplo la construcción, la remodelación o la reparación de la casa, se encontró en un nivel "aceptable” (CJP=52), así como su utilización para el ahorro (CJP=43) 
y para otros usos como el esparcimiento y las emergencias (CJP=57).

El uso de las remesas para la compra de artículos (computadoras, televisores, muebles) se encontró en un nivel "vulnerable" (CJP=38), y el uso de las mismas para algún negocio (tienda, cañaverales, restaurantes, ganado) se ubicó en un nivel "intolerable” (CJP=19) (ver Gráfica 3).

\section{Gráfica 3. Indicador de funcionamientos}

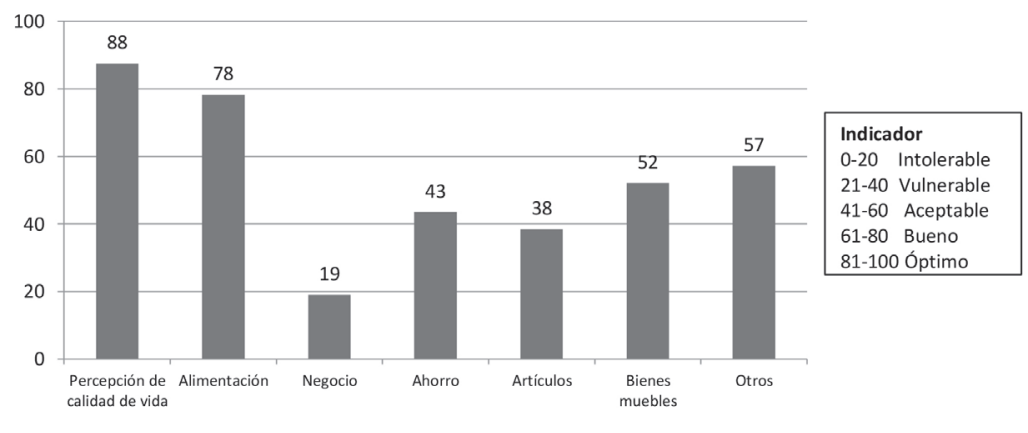

Fuente: elaboración propia.

\section{2. Comparación entre los indicadores de desarrollo humano}

Para que una persona acceda un nivel óptimo de desarrollo humano y para potenciar su libertad y accionar, se requiere, además de que la alimentación esté resuelta, de otros factores que complementen tal proceso. En este sentido, la posibilidad que otorgan ciertos bienes de acceder a otro tipo de habilitaciones, funcionamientos y capacidades, para decirlo en términos senianos, dependerá justamente de su posesión y de lo que el sujeto haga o pueda hacer en función de esto.

Pero además, hay que considerar lo social como un elemento que puede posibilitar u obstaculizar la acción de los individuos, aunque es bien sabido que este tema no 
es la preocupación central del enfoque de capacidades; es decir, su núcleo problemático es más ético que sociológico. Sin embargo, la capacidad de generar funcionamientos y capacidades también depende en cierta medida de los recursos simbólicos y cognitivos de las personas, así como de su capacidad organizacional y su pertenencia o no a una determinada estructura de oportunidades.

Entonces, lo social debe entenderse bajo esta lógica como entorno, pero también como un componente de la acción. Sin este proceso no se puede potenciar la libertad de los individuos para convertir así las capacidades en acciones.

Por tal motivo, el aspecto que interesa evaluar es si las remesas han permitido una mejora en las capacidades y funcionamientos a partir de la comparación de las circunstancias anteriores a la recepción de las remesas (antecedentes de salud y educación), las capacidades (comparación de las remesas en cuanto a salud y educación) y los funcionamientos medidos a partir de la percepción de las personas sobre su propia calidad de vida producto del uso que se le da a las remesas.

Los resultados indican una ligera mejoría con respecto a la percepción que tienen las personas en relación al acceso a la salud, así como la incidencia que esto tiene en su calidad de vida, de ahí que dicho rubro se movió de un nivel "aceptable" (CJP=54) a uno "bueno" (CJP=66). Debe recordarse que la salud es un aspecto fundamental en el enfoque de capacidades y la perspectiva del desarrollo humano.

Para el caso de la educación, los datos son reveladores, pues comparativamente se observa un retroceso a partir de la recepción de remesas, pasando de un nivel "bueno" a uno "aceptable" (de CJP=62 a CJP=56). En cuanto a los funcionamientos, que se refieren a la relación que existe entre el uso de las remesas y si han permitido mejorar la calidad de vida de los familiares de los migrantes, los resultados demuestran que ellos perciben que su calidad de vida se 
ha mejorado considerablemente al ubicarla en un nivel "óptimo" (ver Gráfica 4), aunque refieren que las remesas han permitido contar de manera limitada con bienes materiales, o considerados de lujo, como computadoras y televisores, y sobre todo disponer de ciertos recursos para el ahorro o la inversión en bienes inmuebles, ubicando al uso de las remesas en un nivel "vulnerable".

Gráfica 4. Comparación entre las circunstancias, las capacidades y los funcionamientos

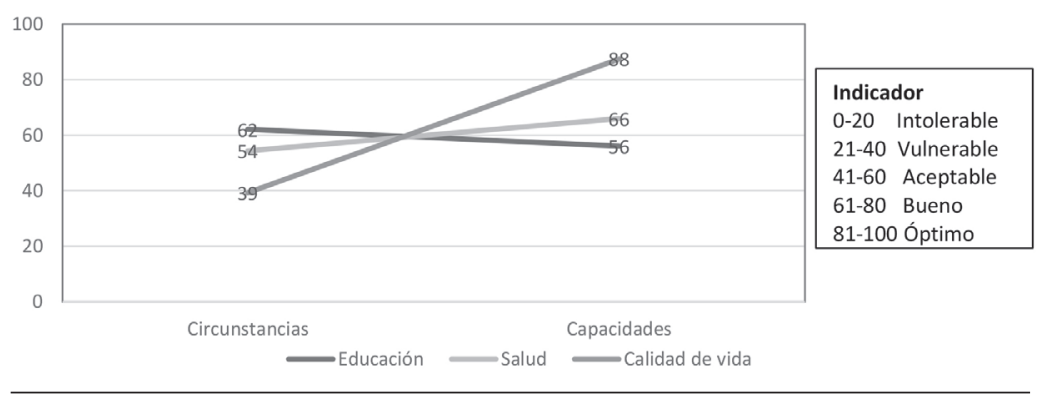

Fuente: elaboración propia.

La Figura 1 presenta los resultados de la matriz de correlaciones producto-momento de Pearson, con el propósito de determinar la relación que existe entre las variables que evalúan una dimensión, y entre las dimensiones.

Entre las variables que determinan cada una de las dimensiones evaluadas, puede apreciarse una relación directamente proporcional entre antecedentes de salud y antecedentes de educación $(\mathrm{r}=0.57)$, los cuales determinan la evaluación de las circunstancias en estos dos ámbitos antes de recibir las remesas.

Del mismo modo, puede observarse una relación positiva entre la comparación de salud y la comparación de educación $(r=0.35)$, las cuales explican las capacidades y, como 
se ha establecido, se refieren a la habilidad para alcanzar los logros a partir de la posesión de recursos.

Por su parte, las variables uso de remesas y calidad de vida están estrechamente relacionadas entre sí $(r=0.50)$. Estas tienen que ver con los funcionamientos, que son los logros que se alcanzan sobre la base de las capacidades que otorgan los recursos y que permiten acceder a mejores niveles de bienestar, siempre en función del incremento de la libertad y la libre determinación de las acciones.

Lo anterior permite constatar que las variables incluidas en cada una de las dimensiones tienen una relación directa y pueden explicar en cierta medida el desarrollo humano a partir de los componentes evaluados.

No obstante, y lejos de la relación que exista entre las variables contenidas en cada una de las dimensiones, la importancia de la correlación producto-momento de Pearson radica en la relación que exista entre las variables. De ahí que pueda apreciarse una relación inversamente proporcional entre los antecedentes de salud y la comparación de salud ( $\mathrm{r}=-0.24)$; lo anterior corrobora lo descrito en el análisis previo, en el sentido que la migración incide positivamente en tanto que las remesas pueden permitir un incipiente incremento en el gasto y potenciar el acceso a la salud (Salgado de Snyder, 1996; Mancillas, 2010; Polanco y Jiménez, 2006; Acosta, et al., 2012).

Del mismo modo, puede apreciarse una relación inversamente proporcional entre los antecedentes de educación y la comparación de la educación $(r=-0.35)$. Algunos estudios, como el de Borraz (2005), han demostrado que la migración, y particularmente las remesas, tienen por lo general un efecto limitado en la educación de niños y jóvenes de aquellas regiones con una población pequeña y con padres con bajos niveles de educación. A ello se debe agregar también la desigualdad, la pobreza y la marginación, como ya se ha dicho. Asimismo, puede ser que la inversión en educa- 
ción en este tipo de contextos no se considere importante o prioritaria, debido al hecho de que existe, por lo general, la percepción de que la educación pública no requiere de una inversión adicional a lo que comúnmente se invierte, es decir, uniformes y útiles, gastos que se realizan independientemente de si se cuenta o no con las remesas.

Los resultados más destacados se encuentran entre las variables de la dimensión capacitación y la dimensión funcionamientos. Puede constatarse que existe una relación alta, positiva y significativa entre la salud y el uso de las remesas $(r=0.51)$, y entre la educación y el uso de las remesas $(r=0.54)$. Esto sugiere que las remesas están incidiendo de manera directa en la primera dimensión, y de manera indirecta en educación a través de la compra de equipos de cómputo y otros artículos varios que sin duda tienen un impacto relativamente positivo sobre esta.

De igual forma, se corroboró una relación directamente proporcional y significativa entre la salud y la calidad de vida $(r=0.51)$, y una relación baja entre la educación y la calidad de vida (0.17). Es decir, en el primer caso las personas sí están percibiendo que ha habido una mejora en su condición de salud, y que esto, en términos generales, repercute en un relativo mejoramiento de su calidad de vida.

Por el contrario, los familiares de los migrantes perciben que las remesas no han permitido mejorar el acceso y los niveles de educación; por esta razón, su percepción es que este aspecto no tiene una incidencia real en el mejoramiento de la calidad de vida. Lo anterior corrobora los hallazgos de cierto sector de la literatura en el sentido de que las remesas sí inciden positivamente en la salud (Salgado de Snyder, 1991; Polanco y Jiménez, 2006), y, por el contrario, que tienen muy baja incidencia en la educación (Borraz, 2005) (ver Figura 1). 
Figura 1. Resultados de la matriz de correlación productomomento de Pearson de las dimensiones del cuestionario de percepción de desarrollo humano

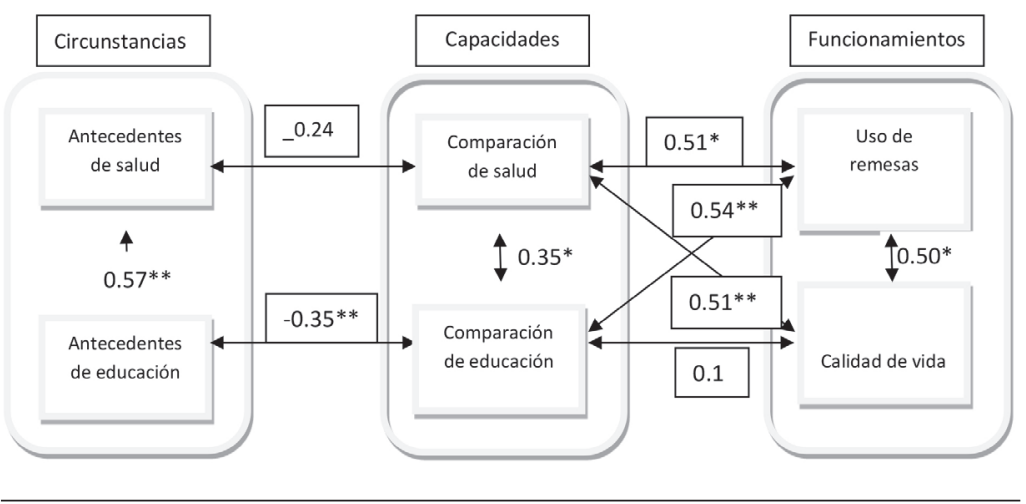

Fuente: elaboración propia.

\section{3. Diferencias entre hombres y mujeres respecto}

a los indicadores de desarrollo humano

La Gráfica 5 presenta los resultados del indicador salud entre hombres, mujeres y el indicador general. Como puede apreciarse, los resultados de las circunstancias entre hombres y mujeres son muy similares: las del primer grupo se encuentran un punto por debajo del indicador general (CJP=53), y las del segundo grupo, un punto por encima de este (CJP=55). No obstante, los resultados de las capacidades reportaron que las mujeres percibieron la resolución de los problemas de salud un punto porcentual por debajo del indicador general, y que los hombres lo percibieron de manera más favorable (CJP=69). Sin embargo, los dos grupos calificaron las circunstancias como "aceptables", y las capacidades se ubicaron en un nivel "bueno". 
Gráfica 5. Comparación del indicador salud entre hombres, mujeres y el indicador general

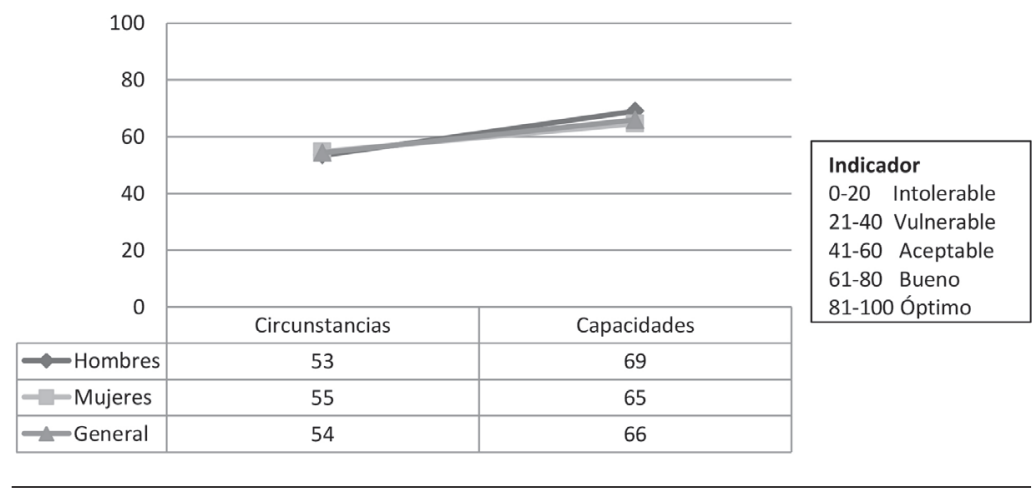

Fuente: elaboración propia.

Los resultados de la comparación del indicador de educación son muy interesantes. A pesar de que tanto las puntuaciones de los hombres, mujeres y el indicador general tienden a ser mejores en las circunstancias que en las capacidades, las mujeres (CJP=66 vs. CJP=59) tienen una mejor percepción en cuanto a su mejoría respecto de los hombres (CJP=53 vs. CJP=49). En este sentido, al evaluar las circunstancias y las capacidades, las mujeres percibieron que la educación pasó de un nivel "bueno" a uno "aceptable", mientras que los hombres percibieron que la educación se mantuvo en un nivel "aceptable" (ver Gráfica 6).

El indicador de calidad de vida es otro aspecto que salta a la vista. En este sentido, los hombres percibieron una mejor calidad de vida, tanto en las circunstancias (CJP=44) como en las capacidades (CJP=92); la percepción de las mujeres se ubicó por debajo del indicador general (CJP=37 vs. CJP=86). Sin embargo, los hombres percibieron que la calidad de vida pasó de un nivel "aceptable" a un nivel "óptimo", mientras que las mujeres percibieron que esta dio un salto más importante, al pasar de un nivel "vulnerable" a uno "óptimo" (ver Gráfica 7). Ya se mencionó que 
Gráfica 6. Comparación del indicador educación entre hombres, mujeres y el indicador general

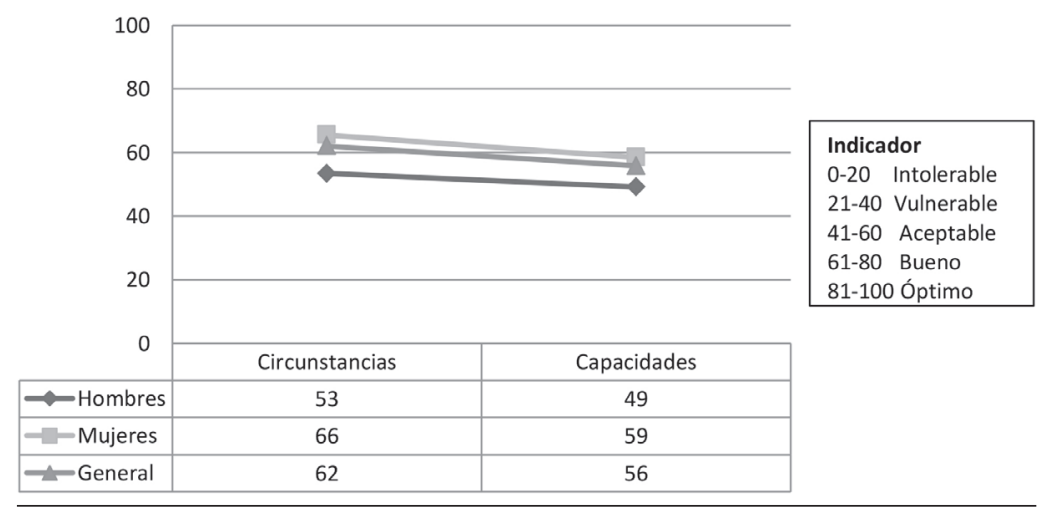

Fuente: elaboración propia.

esta percepción relacionada con la calidad de vida está, en buena medida, influenciada por el hecho de que hay una mayor inversión para gastos de consumo, particularmente la alimentación, por lo que las puntuaciones del indicador general confirman esta tendencia.

Gráfica 7. Comparación del indicador calidad de vida entre hombres, mujeres y el indicador general

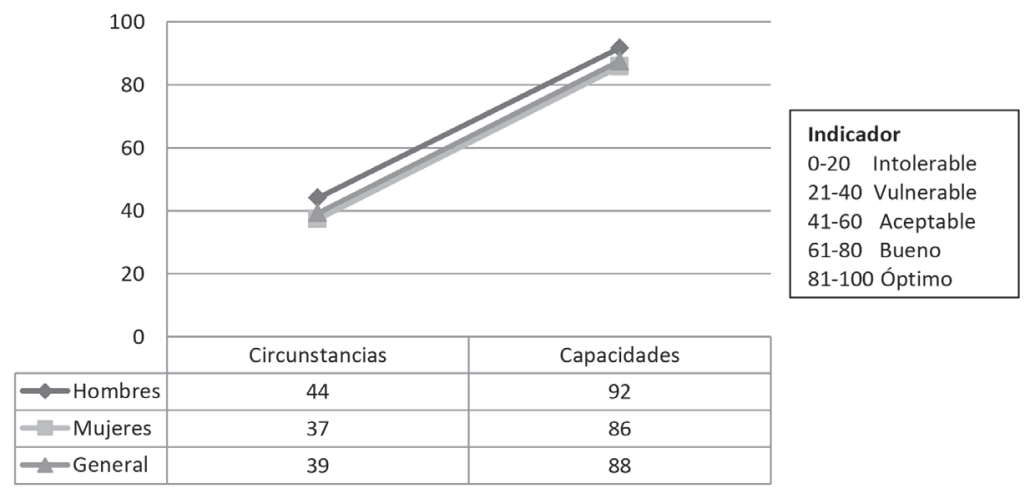

Fuente: elaboración propia. 
Dentro de los resultados de la comparación del indicador funcionamientos entre hombres, mujeres y el indicador general, resulta interesante destacar que los hombres tuvieron una mejor percepción en cuanto al uso que se le dio en un momento determinado a las remesas respecto a las mujeres, y que estas obtuvieron puntuaciones ligeramente por debajo del indicador general. En esta dirección, la alimentación, la posibilidad de abrir un negocio y otros usos se ubicaron un nivel por encima del indicador general ("óptimo", "intolerable” y "bueno", respectivamente) (ver Gráfica 8).

Gráfica 8. Comparación del indicador funcionamientos entre hombres, mujeres y el indicador general

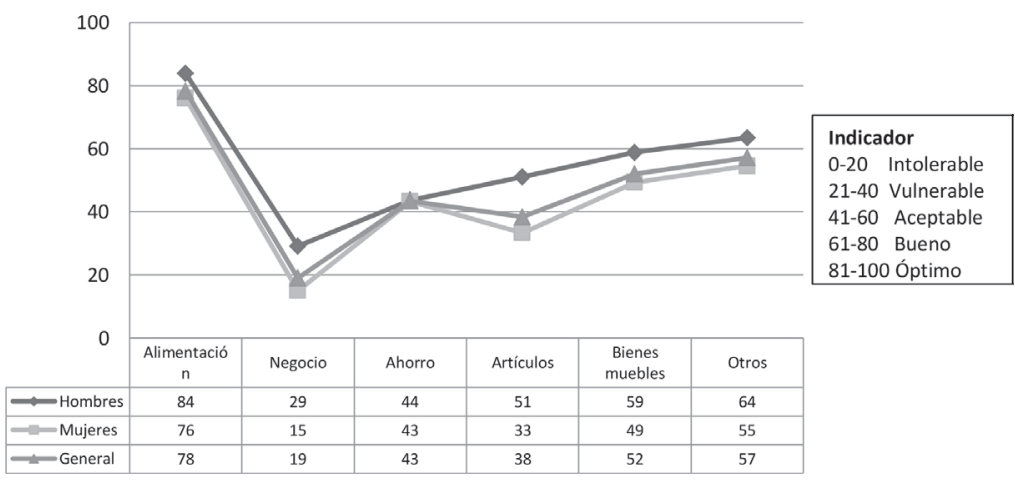

Fuente: elaboración propia.

Asimismo, los resultados del índice de desarrollo humano entre hombres y mujeres confirman la tendencia descrita anteriormente, ya que reportaron una puntuación ligeramente por encima del índice global para el primer grupo (CJP=61), y un resultado por debajo del índice general para el segundo grupo (CJP=59). Sin embargo, esta leve variación ubicó a los hombres en un nivel "bueno" y a las mujeres en un nivel "aceptable." Se puede observar que en general las 
implicaciones de las remesas para el desarrollo de capacidades y la determinación de las acciones resultaron ser positivas, pero no alcanzan los niveles deseables de acuerdo a lo que marcan los estándares del Programa de Naciones Unidas para el Desarrollo (ver Gráfica 9).

Gráfica 9. Índice de desarrollo humano de acuerdo al sexo

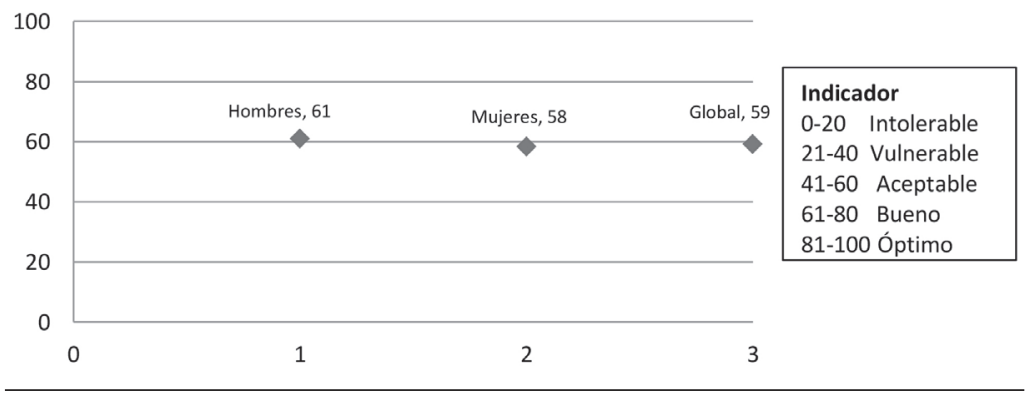

Fuente: elaboración propia.

\section{Conclusiones}

El objetivo de la presente investigación fue evaluar el desarrollo humano de los familiares de los migrantes a partir de la percepción que tienen estos respecto al enfoque de capacidades formulado por Amartya Sen.

En este sentido, los resultados indicaron que la percepción de las personas es medianamente positiva en relación a las condiciones de vida que tenían sobre la base del acceso a la salud y la educación antes de recibir las remesas. No obstante que los datos e indicadores oficiales sobre pobreza y marginación dan cuenta de una realidad caracterizada por grandes rezagos sociales en la región, y particularmente en San José Chiltepec, la percepción que tienen los familiares de los migrantes es relativamente positiva en los rubros antes mencionados. 
Esto puede deberse, por una parte, al hecho de que no son los migrantes en pobreza extrema los que migran, como lo ha señalado la literatura de manera recurrente (Alvarado Juárez, 2008). Por otro lado, la integración de muchas de estas personas a los programas sociales mediante los cuales reciben determinados recursos y servicios puede crear de cierta forma una imagen de que las carencias y los rezagos no son tan agudos; asimismo, el acceso al Seguro Popular de Salud y a la educación pública garantizada pueden ser otros de los factores que moldean esta percepción.

Respecto al indicador de capacidades, pudo apreciarse que estas bajaron con respecto a las circunstancias; se puede establecer que las remesas están impactando de manera indirecta a través de la compra de equipos de cómputo y consumibles, pues los familiares refieren que el dinero se ha utilizado ocasionalmente para estos fines. Además, al ser la educación fundamentalmente pública en la comunidad, las personas no sienten que las remesas estén impactando positivamente en este sentido. Además, pueden existir otros factores que estén propiciando una percepción negativa respecto de la utilidad de las remesas para mejorar la educación.

Para el enfoque del desarrollo humano, la variable ingreso ejerce una función sustancial en el desarrollo de las capacidades, pues permite en teoría acceder a otros bienes y servicios. Además, es uno de los criterios que se utilizan para la elaboración del índice de desarrollo humano. Los resultados que se obtuvieron permiten confirmar los argumentos de un sector importante de la literatura sobre remesas en el sentido de que estas se ocupan primordialmente en el consumo, con énfasis particular en la alimentación (Canales, 2008; García Zamora y Orozco, 2009; Delgado Wise, et al., 2009; Marroni, 2009; Castles y Delgado Wise, 2007).

Si bien es cierto que el consumo es el elemento en el que más están incidiendo las remesas, los resultados a través

\section{6}


de las calificaciones jerárquicas porcentuales muestran que para los familiares de los migrantes este aspecto es el que tiene el mayor impacto en la calidad de vida. Más allá de la educación, la salud y la inversión productiva, las personas consideran que la alimentación ocupa el primer lugar entre los aspectos que contribuyen a mejorar sus condiciones de vida. La gran mayoría lo considera así por el hecho de que resolver adecuadamente el tema de la alimentación no estaba totalmente garantizado antes de migrar el familiar $y$, por ende, ellos perciben que repercutía negativamente en su calidad de vida.

Esto nos remite a plantear inicialmente que los ingresos por concepto de remesas han logrado incrementar la calidad de vida de los familiares de los migrantes en esta comunidad, aunque no de una manera óptima pues la percepción general sobre el bienestar está condicionada por la idea de que la inversión en alimentación se traduce en una mejora sustancial de la calidad de vida de las personas.

Aun reconociendo que la alimentación es una necesidad que está por encima de otras, lo cierto es que la posesión de ciertos bienes, la recreación e inclusive el ahorro pueden tener también una incidencia directa en el mejoramiento de la calidad de vida y permitir a su vez el acceso a otro tipo de bienes o recursos. Piénsese, por ejemplo, en la compra de ciertos artículos, como computadoras. Estas se podrían convertir en una herramienta muy útil para los hijos o las personas que estén estudiando, pues permitirían incrementar sus habilidades y contribuir así en su proceso formativo. En este caso, sí se cumpliría la premisa que plantea el enfoque de capacidades de que la posesión de bienes y recursos necesariamente debe conducir a un mejoramiento y ampliación de las capacidades, y con ello incrementar los niveles de bienestar de las personas.

Sin embargo, de acuerdo a los resultados, los ingresos por remesas no están impactando de forma significativa los 
demás rubros. Esto puede deberse a varios motivos: primero, el hecho de que para la gran mayoría de los involucrados esta es la única fuente de ingresos o la que proporciona la mayor entrada de recursos económicos, por lo que es difícil que pueda subsanar todas las necesidades que tienen estas personas; en segundo lugar, puede deberse a la frecuencia y el monto de los envíos, que generalmente se realizan de tres a cinco veces por año (Acosta, et al., 2009, p. 182).

Además de lo dicho en líneas anteriores, hay que tener en cuenta el contexto social en el que se ubican estas personas, que, como ya se ha visto, está caracterizado por una gran desigualdad en el acceso a oportunidades, con grandes rezagos y con altos niveles de marginación y pobreza. Esta situación influye de manera directa en la calidad de vida de las personas y se convierte, desde luego, en una barrera que limita las posibilidades de superación de este estado de las cosas, puesto que existe un problema mayor que es la deficiente estructura de oportunidades que ha generado un gradual proceso de exclusión.

Entonces, es hasta cierto punto natural que en un ambiente de carencias y permanentes privaciones, las personas le asignen un peso específico a la satisfacción de una necesidad básica como la alimentación, y que esto a su vez determine en buena medida la consideración de que la resolución adecuada de esto sea sinónimo de bienestar para las personas con este tipo de antecedentes o circunstancias de vida. Esto no quiere decir que se le reste importancia al peso específico que tienen las remesas para paliar las necesidades de alimentación, y que esto sea visto como sinónimo de bienestar, sino que se hace hincapié en que los funcionamientos y capacidades, como punta de lanza del desarrollo humano, no dependen solamente de la alimentación o posesión de recursos, sino de lo que las personas puedan hacer con ellos.

\section{8}


En cuanto a los resultados de la comparación entre las circunstancias, las capacidades y los funcionamientos, se aborda en principio lo respectivo a la salud. Para el enfoque de capacidades, esta es un medio y un fin: un medio para el logro del bienestar común y un fin como elemento sustantivo para el desarrollo humano (Sen, 1995).

Pero la salud no sólo está en riesgo cuando las personas son amenazadas por enfermedades, sino que múltiples aspectos de su cotidianidad y espacio vital también constituyen peligros para el desarrollo de todas sus potencialidades. En ese sentido, la pobreza, la violencia, la carencia de alimentos y trabajo, la inseguridad laboral, la falta de acceso a agua potable, una vivienda inadecuada, y el analfabetismo, entre otras violaciones de los derechos humanos, constituyen una amenaza para la salud de las personas y las colocan en una posición de vulnerabilidad frente a esos riesgos. La literatura especializada ha planteado que la migración puede tener múltiples efectos sobre la salud del migrante y de sus familiares (Salgado de Snyder, 1996).

Regresando a las puntuaciones resultantes, y observándolas en perspectiva comparada, se tiene que la diferencia porcentual no es tan amplia con respecto a la situación experimentada antes de recibir el dinero. Llama la atención que a pesar que este aspecto no es una de las prioridades a la hora de utilizar esos recursos, las personas tienen una opinión medianamente positiva respecto al impacto que tienen estos recursos en la salud. Esto puede deberse igualmente al limitado acceso y uso de los servicios de salud antes de recibir las remesas, y, en general, a la condición sociodemográfica en la que están insertas estas personas, lo que ciertamente puede condicionar la percepción en el sentido de sobrevalorar su condición actual frente a otra en la que las carencias eran la norma.

Asimismo, la emergencia de programas sectoriales como el Seguro Popular, como ya se ha mencionado, de cierta 
forma puede condicionar a las personas a creer que la accesibilidad ha mejorado, y con ello su propia condición de salud, aunque en la práctica no reciban ningún tipo de servicio o atención médica, pues la sola idea de contar con un servicio de esta naturaleza puede incidir sobre la percepción.

En lo tocante a la educación, las respuestas refieren que con la entrada de estos recursos se ha experimentado un impacto negativo en este rubro, no solamente en la educación básica, sino en general. Nuevamente el contexto puede ser determinante en la generación de dicha percepción, pues hay que tener en cuenta el contexto de la región y las circunstancias de vida de los sujetos involucrados.

Otra de las razones puede ser la deserción escolar, cuyos orígenes se ubicarían en la reprobación, la falta de incentivos, y hasta en la propia cultura migratoria, que comúnmente produce estereotipos que tienden a ser copiados o reproducidos por los jóvenes para tratar de insertarse en una dinámica similar. En estos casos, la opinión respecto al impacto que tienen las remesas en la educación estaría mediada por estos aspectos.

En particular, se considera que la mayoría de los elementos antes mencionados tienen que ver con la escasa ponderación que se le asigna al papel de las remesas como impulsoras de la educación en los familiares de los migrantes de San José Chiltepec. Los datos socioeconómicos oficiales que se presentaron en un apartado anterior permiten sostener este argumento, pues los rezagos de todo tipo siguen prevaleciendo y coinciden con la apreciación que la gente tiene en este sentido.

Se puede establecer con toda claridad que si la educación no ha sido impactada de manera positiva por las remesas, es de suponerse que la calidad de vida se verá impactada, pues según Sen (1995) esta es fundamental para potenciar habilitaciones y capacidades. 
A ello debe agregarse la posibilidad de actuar y decidir con base precisamente en esos requerimientos antes citados, los que hipotéticamente se verían afectados de manera negativa. Es decir, si las acciones están orientadas hacia la consecución de un fin, y si estas se ven afectadas por una insuficiente habitación y capacidad, entonces la resolución de una situación no deseada o poco satisfactoria tendrá mínimas posibilidades de resolverse. Es en este sentido que la teoría de la acción establece cierta coincidencia con el enfoque de capacidades, pues permite asociar la idea de libertad con la capacidad de acción de los individuos.

En referencia a la comparación entre hombres y mujeres respecto al indicador de salud, los resultados reportaron pequeñas diferencias. Lo anterior puede deberse al hecho que las mujeres tienen una mayor capacidad de respuesta a los problemas de salud, lo que influye en su decisión de buscar asistencia médica ante un problema específico. Además, estudios sobre el acceso a servicios de salud han identificado diversos factores estructurales que tienen especial repercusión en la búsqueda y utilización de servicios de salud por parte de las mujeres (Nash-Durrenda, 1992; Barraza Lloréns, et al., 2002). Entre estos factores se encuentran su condición económica, la confianza en el sistema de atención médica y la representación del proceso salud-enfermedad, entre otros.

En el caso de México, se ha documentado que las mujeres tienden a buscar atención médica antes, y con mayor frecuencia, que los hombres (Kaijzer, 2003). Todos estos elementos pueden favorecer el hecho de que las mujeres tengan una valoración más positiva que los hombres respecto al impacto que tienen las remesas en la salud.

En el caso del indicador de educación, ya se comentó anteriormente que puede influir en esta percepción el gasto tradicional que se hace en educación pública, que puede provocar que la opinión de las personas en relación 
al impacto que tienen las remesas se considere como de poca importancia. Sin embargo, nuevamente son las mujeres quienes tienen las mejores valoraciones. Esto lleva a pensar que a pesar de que la incidencia puede considerarse negativa, estos efectos son menores en las mujeres, debido probablemente a una menor deserción escolar y a una mayor cantidad de años de estudio para este sector.

Finalmente, las diferencias encontradas en el indicador de funcionamientos pueden deberse al hecho de que los hombres perciben que sí ha habido un mejoramiento en sus condiciones de vida, por el hecho de que su familiar haya migrado, respecto a las que tenían en el pasado. En cambio, las mujeres tienen una percepción menos favorable. Esto puede deberse a que son ellas las que regularmente administran el dinero y tienen por ello una idea más clara de los gastos y su distribución, así como de los aspectos en los que este tiene una mayor incidencia positiva; es por ello que su percepción es ligeramente diferente a la de los hombres.

Los resultados que aquí presentamos confirman de cierta forma los argumentos enunciados por Amartya Sen en el sentido de que la sola posesión de recursos no es garantía para que las personas accedan a grados mayores de desarrollo humano, y de que el contexto e historia de vida de las personas determinan en buena medida el uso que se le da a los mismos, pero también las posibilidades de que los sujetos se conviertan en agentes de su propia transformación, es decir, con capacidades y habilitaciones que les permitan llevar acabo la vida que quieren vivir.

A partir de la evidencia recabada, se pudo establecer que los supuestos beneficios que propicia la migración, de los cuales dan cuenta los informes mundiales del 2007 y 2009, no son tan significativos, pues las remesas son utilizadas casi en su totalidad para financiar el consumo (alimentación y compra de enseres domésticos). 
A pesar de que el consumo tiene una relación con el desarrollo, no puede considerarse como el único factor que lo garantiza, pues se requiere, además, del acceso a otros bienes, recursos y servicios. Así, pues, se reconoce que el consumo tiene un impacto en la generación de funcionamientos y capacidades, pero no es decisivo en la transformación de las circunstancias de vida de los individuos. Por lo tanto, su incidencia es medianamente positiva y no puede considerarse como un elemento que cambie la vida de las personas en un sentido más amplio.

Asimismo, más allá que la relación remesas-desarrollo humano es por demás compleja, sus impactos en comunidades con altos niveles de pobreza y marginación son relativos, pues prevalecen en dichas comunidades condiciones cuya superación no depende del envío de dinero que realizan los migrantes. Si bien es cierto que hay efectos positivos, estos recursos sirven por lo general para paliar necesidades básicas y elementales como la alimentación y la salud, que si bien son palancas que impulsan el desarrollo, para el caso en cuestión no se constituyen como un elemento central.

El desarrollo, siguiendo a Dubois, se debe entender como un proceso de transformación de los individuos y la sociedad, que se mueve desde viejas formas de pensamiento y organización social hacia otras nuevas (Dubois, 2008). Es un proceso de cambio dinámico, que afecta no sólo a los modelos de producción y a la tecnología, sino también a las instituciones sociales, políticas y económicas, impactando los niveles de desarrollo humano.

Fecha de recepción: 11 de septiembre de 2014

Fecha de aceptación: 25 de enero de 2016 
Bibliografía

Acosta Uribe, B., Yamel Flores Yeffal, N., y Ruiz Sánchez, J. (20I2). La salud mental de las mujeres ligadas a la migración: El caso de San José Chiltepec, Oaxaca. México: ECOSUR-El Colegio de la Frontera Norte-El Colegio de Michoacán-CIESAS.

- (2009). "Las remesas internacionales y su incidencia en el desarrollo humano en San José Chiltepec, Oaxaca”. Nova Scientia, (3), 167-187.

Aguilar-Morales, J. E., Vargas Mendoza, J. E., Peralta Reyes, E. B., et al. (2008). "Migración, salud mental y disfunción familiar: Impacto socioemocional en la familia del indígena oaxaqueño migrante". Centro regional de investigación en psicología, 2(I), 5I-62.

Alvarado Juárez, A. M. (2008). "Migración y pobreza en Oaxaca”. Revista El Cotidiano, (148), 85-94.

Barraza-Lloréns, M., Bertozzi, S., González-Pier, E., y Gutierrez, J. P. (2002). "Adressing inequity in health care in Mexico". Health Affairs, (2I), 47-56.

Borraz, F. (2005). "Assessing the Impact of Remittances on Schooling: the Mexican Experience". Global Economy Journal, 5(I), I-30.

Canales, A. I. (2008). Vivir del norte. Remesas, desarrollo y pobreza en México. México: Consejo Nacional de Población.

Castles, S., y Delgado Wise, R. (2007). Migration and Development: Perspective from the south. México: Universidad Autónoma de Zacatecas-OIM.

Cejudo Córdoba, R. (2007). "Una aproximación a la teoría de Amartya Sen”. Revista Internacional de Sociología, $\operatorname{LXV}(47), 9-22$.

CONAPO (2005). Compendio estadístico Oaxaca 2005 y 2010. México: CONAPO.

Delgado Wise, R., Márquez Covarrubias, H., y Rodríguez Ramírez, H. (2009). "Seis tesis para desmitificar el

\section{4}


nexo entre migración y desarrollo". Revista Migración y Desarrollo, (I2), 27-52.

Hurtado Arriaga, G., Rodríguez Contreras, V., Escobar Torres, J., Santamaría Suárez, S., y Pimentel Pérez, B. M. (2008). "Los que se quedan. Una experiencia de migrantes”. Revista científica electrónica de psicología, (6), 9-28. García Zamora, R., y Orozco, M. (coord.) (2009). Migración internacional, remesas y desarrollo local en América Latina y el Caribe. México: Universidad Autónoma de ZacatecasMiguel Ángel Porrúa.

Kaijzer, B. (2003). "El género y el proceso salud-enfermedad-atención”. Gén Sal, I, 3-7.

Mancillas Bazán, C. (2010). Salud mental e interpersonal en parejas migrantes mexicanas. Seminario permanente sobre migración internacional, migración y salud mental. México: El Colegio de México.

Marroni, M. G. (2009). Frontera perversa, familias fracturadas. Los indocumentados mexicanos y el sueño americano. Puebla: Instituto de Ciencias Sociales Alfonso Vélez Pliego.

Morales, M. L. (2005). Psicometría aplicada. México: Editorial Trillas.

Moreno Pérez, S. (2008). Migración, remesas y desarrollo regional en México. México: Centro de Estudios Sociales y de Opinión Pública.

Nash-Durrenda, G. (1992). "Women access to health care in developing countries". Soc Sci Med, 35(4), 6I 3-6 I7. OIM (200I). Informe sobre las migraciones en el mundo, año 2000. S/d: Organización de Naciones Unidas.

PNUD (1990). Concepto y medición del desarrollo humano. Nueva York: Programa de Naciones Unidas para el Desarrollo/Palgrave-Macmillan.

(2007). Informe sobre desarrollo humano 2007. Migración y desarrollo humano. Nueva York: Programa de Naciones Unidas para el Desarrollo/Palgrave-Macmillan.
Bibliografía 
Bibliografía 